\title{
MUSCULOSKELETAL MANIFESTATIONS IN DIABETIC PATIENTS AT A TERTIARY CENTER
}

Timóteo Quereza e Silva Faria1,*, Sandra Lúcia Euzébio Ribeiro¹, Julia de Oliveira Simão¹, Ana Beatriz Vieira da Silva ${ }^{1}$, Bárbara Letícia da Costa Corrêa ${ }^{1}$, Gabriel de Souza Araújo ${ }^{1}$, Lívia Maria Alves Rebouças Tomé Praciano ${ }^{1}$, Fernanda Lemos Magalhães ${ }^{1}$, Paulo Vítor de Amorim Silva ${ }^{1}$, Igor Lorenzo Ribeiro de Oliveira ${ }^{1}$, Anny Karolinny Ferreira da Silva ${ }^{1}$, Angela Santos Veras de Souza ${ }^{1}$, Alana Nogueira da Silva ${ }^{1}$, Ana Beatriz Vieira da Silva ${ }^{1}$, Ana Paula Narciso Resk Maklouf ${ }^{1}$, Victória Costa de Araújo ${ }^{1}$

1.Universidade Federal do Amazonas, Manaus (AM), Brazil.

*Corresponding author: timoteoquereza@ufam.edu.br

\section{BACKGROUND}

Diabetes mellitus (DM) is a chronic metabolic disease with high morbidity and mortality. According to a WHO survey (2018), 16 million Brazilians had DM, which increased the incidence rate in 10 years by more than $60 \%$. Brazil is in 4 th place among the countries with the highest incidence. It is considered a global epidemic, associated with renal and ophthalmologic disturbances derived from the metabolic alteration caused by DM type 1 and 2. Diabetes mellitus is associated with a great variety of musculoskeletal manifestations, many of them subclinical, and correlated with time of evolution and inadequate control of the disease, and they must be recognized and adequately treated.

\section{METHODS}

A cross-sectional and descriptive study was carried out, where clinical triage was performed at an endocrinology outpatient clinic, where patients with musculoskeletal complaints were identified. Patients with pain complaints were referred for evaluation with a rheumatologist, and clinical, laboratory and imaging investigations were performed for the diagnosis of rheumatic disease. A survey with epidemiological and clinical data was applied. In this research, the main musculoskeletal manifestations found in patients with diabetes mellitus are discussed.

\section{RESULTS}

In our study with 240 patients diagnosed with DM1 and DM2 were interviewed with a mean age of $53 \pm 15.09$ years, being 180 (75\%) female and 60 (25\%) male. There were 193 (80.4\%) patients with type 2 DM and 47 (19.6\%) with type 1DM; of these, 173 (72\%) presented musculoskeletal complaints that influenced their quality of sleep or work. Of patients with musculoskeletal pain, 85 (49\%) were younger than 60 years old. The musculoskeletal pathologies found associated with DM were: rheumatoid arthritis (27.8\%), osteoarthritis (25.9), fibromyalgia (22.2\%), osteoporosis (20.4\%), lupus (11.1\%), psoriatic arthritis (9.3), trigger finger, bursitis and gout (7.4\% each). The numbers of lupus patients may be justified due to corticosteroid-induced hyperglycemia of constant use.

\section{CONCLUSION}

Diabetes mellitus is directly related to musculoskeletal manifestations in more $70 \%$ of patients. These correlation data are obtained through epidemiological studies since the pathophysiological mechanisms of this association are not well elucidated. Identification with early diagnosis and subsequent early treatment bring improvement in quality of life to these patients. The study and establishment of the parameters of this relationship can be used in the future as an adjunct in the early identification of DM in patients not yet recognized as such.

\section{KEYWORDS}

Diabetes mellitus, Dupuytren’s contracture, Trigger finger, Bursitis, Carpal tunnel syndrome. 